\title{
A ATUAÇÃO DO ENFERMEIRO INTENSIVISTA PARA PACIENTES EM HEMODIÁLISE
}

\section{ARTIGO DE REVISÃo}

TONINI, Paula Silva Santos ${ }^{1}$

TONINI, Paula Silva Santos. A atuação do enfermeiro intensivista para pacientes em hemodiálise. Revista Científica Multidisciplinar Núcleo do Conhecimento. Ano. 06, Ed. 08, Vol. 04, pp. 118-130. Agosto 2021. ISSN: 2448-0959, Link de acesso: https://www.nucleodoconhecimento.com.br/saude/pacientes-em-hemodialise, DOI: 10.32749/nucleodoconhecimento.com.br/saude/pacientes-em-hemodialise

\section{RESUMO}

A Unidade de terapia intensiva é uma área destinada a internação de pacientes graves. O enfermeiro intensivista deve dominar a fisiologia para planejamento da assistência individualizada ao paciente crítico. A insuficiência renal é uma complicação recorrente em pacientes grave, de etiologia pré-renal, intrínseca ou pós-renal, sua classificação pode ser de acordo com dois guide lines diferentes: KDIGO ou AKIN. Este artigo tem como pergunta norteadora: qual deve ser a atuação do enfermeiro intensivista na assistência ao paciente em hemodiálise e quais os riscos apresentados para o paciente e profissionais? Temos como objetivo identificar os principais pontos a serem observados pelo enfermeiro intensivista durante realização da Terapia de Substituição Renal (TRS) de emergência, descrever quais os riscos envolvidos para o paciente, o profissional, e equipe envolvida na assistência. Para atender ao objetivo, foi realizada uma revisão da literatura. As bases de dados consultados foram SciELO, Google Acadêmico, Klinical Key, Lilacs e Ministério da Saúde, utilizando os descritores: "insuficiência renal aguda"; "injuria renal"; "hemodiálise em unidade de terapia intensiva"; "papel do

\footnotetext{
${ }^{1}$ Bacharel em enfermagem, pós-graduada em Enfermagem Intensiva especialista em oncologia.

RC: 94364

Disponível em: https://www.nucleodoconhecimento.com.br/saude/pacientes-emhemodialise
} 
enfermeiro em unidade de terapia intensiva". Foram coletados materiais publicados no período de 2010 a 2020. Os resultados encontrados foram monitorização dos marcadores biológicos, balanço hídrico rigoroso, tratamento de causas reversíveis, intervenção dietética e hemodiálise. Foram identificadas complicações durante a hemodiálise como hipotensão, caibas, náusea, cefaleia, dor torácica, angina prurido, hipotensão, hipertermia, além de cuidados com cateteres e diferentes tipos de anticoagulações. Foram apontados riscos ocupacionais por manipulação de material biológico, pérfuro-cortantes e riscos ergonômicos para profissionais; riscos de trombose, sepse, entre outros, para pacientes. Conclui-se que o enfermeiro deve buscar constante aprimoramento e atualização profissional, sua atuação interfere diretamente no prognóstico do paciente, suas ações e conhecimento refletem e impulsionarem o trabalho da equipe, além de proporcionar um vínculo entre profissionais, paciente e familiares.

Palavras-Chave: Insuficiência renal aguda, Hemodiálise, Enfermeiro Intensivista, Unidade de Terapia Intensiva.

\section{INTRODUÇÃO}

A resolução № 7 de Fevereiro de 2010, do Ministério da Saúde, define Unidade de Terapia Intensiva (UTI) uma área crítica destinada à internação de pacientes graves, que requerem atenção profissional especializada de forma contínua, além de materiais específicos e tecnologias necessárias ao diagnóstico, monitorização e terapia, necessita de requisitos mínimos para seu funcionamento, infraestrutura física, organização, recursos humanos e assistenciais, exige a atuação de profissionais especializados e atualizados, envolvidos num processo permanente de aprendizagem. (MINISTÉRIO DA SAÚDE, 2010).

Dentre os profissionais atuantes em UTI, enfermeiro intensivista é responsável por planejar a assistência ao paciente crítico. Esta assistência individualizada é formulada por meio da Sistematização do Processo de Enfermagem (SAE), uma 
ferramenta utilizada exclusivamente pelo enfermeiro para gerenciar cuidados e nortear toda a equipe de enfermagem para continuidade do cuidado incluindo enfermeiros, técnicos e auxiliares. Concebido através de cinco fases: histórico de enfermagem que se dá através de coleta de dados, diagnóstico, planejamento, implementação e avaliação de enfermagem, a acurácia deve permear todas as fases da avaliação, as decisões e o processamento de informações que geram os diagnósticos, embasam a comunicação e as decisões para a alcançar os resultados esperados. Assim atuação do enfermeiro é indispensável para um bom prognóstico no cuidado intensivo (GRASSI et al., 2017).

O domínio da fisiologia é citado por Barbosa et al. (2012) como uma atribuição fundamental do enfermeiro neste cenário para a manutenção da homeostasia. Alguns órgãos nobres requerem mais atenção, necessitam de monitorização contínua, como o sistema respiratório, hemodinâmico, neurológico gastrointestinal, hepático e função renal. Todos os sistemas são de grande importância, e seu equilíbrio garante a sobrevivência do paciente. Dentre estes sistemas, alguns merecem monitorização contínua, pois afetam rapidamente os demais sistemas afetando a homeostasia é o caso do sistema renal, os rins são órgãos extremamente sensíveis, e importantes para eliminação de substâncias tóxicas produzidas pelo organismo, controlam a pressão arterial a longo prazo, regulam a osmolaridade e o potencial hidrogeniônico $(\mathrm{pH})$ do meio extracelular, interferem no metabolismo ósseo, estimulam a produção de glóbulos vermelhos e participam dos mecanismos de neoglicogênese.

A Injúria Renal Aguda (IRA) é uma complicação recorrente grave com múltiplas etiologias, está relacionada a taxa de mortalidade e tempo de internação prolongado, acomete diferentes níveis de atendimento à saúde, podendo chegar à $20 \%$ dos pacientes internados em UTI, estima-se ainda que 10\% destes, necessitem de tratamento com terapia renal substitutiva (hemodiálise). Desta forma é imprescindível a qualificação do profissional enfermeiro, atento à fatores de risco, diagnóstico precoce, bem como preparo da infraestrutura e manejo dos 
equipamentos para realização segura e eficaz dos procedimentos hospitalares (NASCIMENTO et al., 2016).

Ponce et al. (2011) descreve IRA como uma súbita queda de filtração glomerular, acompanhada de retenção de resíduos nitrogenados e distúrbios hidroeletrolíticos, complexa e sem consenso em sua definição. Sua etiologia pode ser:

- Pré-renal: resultante da hipoperfusão renal devido a diminuição do volume intravascular efetivo, com consequente desidratação, vasodilatação periférica, ou por um baixo débito cardíaco, é o tipo mais comum de IRA. Insuficiência renal;

- Itrínseca: decorrente de uma variedade de lesões aos vasos renais, glomerulares tubulares, ou intersticiais, normalmente causadas por toxicidade, reações imunológicas de forma idiopática, iatrogenias, ou parte de uma doença sistêmica ou renal primária;

- Pós-renal: resulta de uma obstrução do trato urinário alto ou baixo. Para uniformizar o diagnóstico e a classificação desta síndrome, alguns critérios adotados são alterações agudas dos níveis séricos da creatinina, aumento superior a $0,3 \mathrm{mg} / \mathrm{dL}$ ou relativo de $50 \%$ em relação ao valor basal, e débito urinário (oligúria) diminuição inferior a $0,5 \mathrm{ml} / \mathrm{kg} / \mathrm{min}$ por mais de 6 horas.

O conjunto destas manifestações são avaliados e identificados de acordo com a guide line KDIGO (Clinical Practice Guideline for Acute Kidney Injury) e classificados como KDIGO 1, 2 ou 3, quanto maior a classificação, menor função renal, outro guide line para sistema de classificação comumente utilizado é AKIN (Acute Kidney Injury Network) tem como diretriz indicadores dos estágios de acometimento renal, listados em estágios 1-risco, 2-lesão renal, 3-falência renal. O é prognóstico grave, porém há evidências da queda da mortalidade nos últimos anos, diversos fatores contribuem para isso, como diferentes técnicas e tecnologias dialíticas, identificação de fatores de risco e o diagnóstico precoce. 
Em vista dos problemas decorrentes da insuficiência renal, que requer diferentes cuidados em seus diferentes estágios, especialmente quando já se encontra em Terapia de Substituição Renal (TRS) normalmente conduzida por enfermeiros em unidade de terapia intensiva para pacientes em estado grave, surgiram as perguntas norteadoras deste trabalho: qual deve ser a atuação do enfermeiro intensivista na assistência ao paciente em hemodiálise e quais os riscos apresentados para o paciente e profissionais? O objetivo deste trabalho é realizar uma revisão da literatura, identificando os principais pontos a serem observados pelo enfermeiro intensivista durante realização da TRS de emergência, descrever quais os riscos envolvidos para o paciente, o profissional, e equipe envolvida na assistência. A importância deste trabalho justifica-se por nortear profissionais intensivistas no cuidado à pacientes em estado grave com IRA em hemodiálise, norteando os principais pontos de atenção, para desta forma melhorar o gerenciamento da assistência prestada, minimizando riscos ao paciente e equipe.

Foi realizada pesquisa bibliográfica por uma revisão analítica de literatura sobre a importância dos cuidados de enfermagem prestados a pacientes críticos em hemodiálise e insuficiência renal aguda em unidade de terapia intensiva. As bases de dados consultadas foram SciELO, Google Acadêmico, Klinical Key, Lilacs e Ministério da Saúde, utilizando descritores como, "insuficiência renal aguda", "injuria renal", "hemodiálise em unidade de terapia intensiva", "papel do enfermeiro em unidade de terapia intensiva" publicados nos últimos dez anos. O levantamento bibliográfico ocorreu durante os meses de maio a setembro de 2020, onde critérios de inclusão foram artigos publicados durante o período de 2010 a 2020, no idioma português. Exclui-se artigos anteriores à 2010, artigos com temas sem relevância para o estudo, e artigos sem resumos. A partir dos critérios utilizados foram encontrados 40 artigos, dentre os quais, após avaliação de acordo com a temática proposta, foram selecionados 12 artigos para leitura na íntegra. 


\section{DESENVOLVIMENTO}

De acordo com o Manual de Diálise da Nefroclínica (2012) o tratamento da IRA consiste em possibilitar os rins debilitados manter a homeostasia, ao passo que possa pesquisar e tratar todos os fatores que contribuem para o problema. A deterioração da função renal requer intervenção dietética e controle da ingesta alimentar, primordialmente consumo de proteínas e líquidos assegurando uma ingesta calórica adequada; um rigoroso acompanhamento dos níveis séricos bioquímicos particularmente ureia, creatinina e $\mathrm{pH}$, mensurado no mínimo uma vez a cada vinte e quatro horas, acompanhamento do balanço hídrico através de um fidedigno registro dos ganhos e perdas diários.

Em caso de piora clínica, prioriza-se o tratamento com hemodiálise, a fim de substituir a função renal temporariamente, visando manter a homeostasia. A equipe de enfermagem atua diretamente nesse processo, principalmente na resolução de possíveis complicações que podem ocorrer durante o tratamento, evidenciando a importância do enfermeiro intensivista com conhecimento em diálise. Neste curso é primordial que o enfermeiro conheça individualmente o paciente, enfatizando a importância dos processos de enfermagem, assim como o diagnóstico e processos patológicos, viabilizando o cuidado e reabilitação do paciente, envolvendo familiares visando um cuidado holístico (LEONARDI, 2019).

Schwanke et al (2018) relata que a terapia renal substitutiva requer um acesso venoso calibroso, a qualidade de vida e mesmo a sobrevivência destes pacientes dependem da performance deste acesso venoso. Os sítios de punção incluem veias jugulares internas e subclávias consideradas como opção inicial, veias femorais como alternativa, contudo a permanência de cateteres de duplo lúmen por tempo prolongado associa-se frequentemente a trombose venosa, infecção ou outras complicações influenciando diretamente na escolha do sítio de inserção deste cateter. 
Sousa (2015) relata que no decorrer da hemodiálise podem ocorrer complicações frequentes que requerem uma rápida identificação e atuação do enfermeiro como:

- Hipotensão devido a velocidade ou flutuações da ultrafiltração, hipovolemia, medicamentos anti-hipertensivos, superaquecimento da solução dialítica, ingesta alimentar, disfunção diastólica, Intervenções como posição de Trendelemburg, administração de volume, alterações da velocidade de filtração, ou diminuição de perdas são responsabilidade da enfermagem.

- Cãibras Musculares decorrentes de hipovolemia abrupta, estão intimamente relacionados com a hipotensão, é mais comum em pacientes desidratados ou em uso de soluções dialíticas pobre em sódio, a prevenção das cãibras é evitar hipotensão durante a hemodiálise, e o tratamento está relacionado a soluções hipertônicas como soro fisiológico, glicosado e/ou associação de gluconato de cálcio.

- Náuseas e vômitos têm etiologia multifatorial, porém está estreitamente ligado a hipotensão, pode ser indicativo da síndrome do desiquilíbrio. Deve-se otimizar a administração de antieméticos, outra prevenção é diminuir o fluxo sanguíneo na primeira hora de hemodiálise, aumentando progressivamente no decorrer do tratamento.

- Cefaleia de etiologia desconhecida, pode proceder uma síndrome do desequilíbrio, resultar de hipotensão, ou ainda da diminuição dos níveis séricos de cafeína uma vez que a hemodiálise depura grande parte desta substância, o tratamento pode ser administração de analgésicos, e redução do fluxo sanguíneo na primeira hora de hemodiálise, aumentando gradativamente nas horas subsequentes.

- Dor torácica e/ou dor lombar é pouco comum, e sua causa desconhecida, porém há teorias que indicam uma resposta imunológica, que se resolve com a substituição da membrana utilizada por membrana sintética ou celulose substitutiva. 
- Angina é comum e está correlacionado a hemólise, hipovolemia e diminuição do débito cardíaco que ativa a liberação de epinefrina, induzindo a vasoconstrição coronária, a prevenção é evitar a hipovolemia.

- Prurido é um sintoma comum causado pela elevação da uremia por ter um efeito tóxico sobre a pele, normalmente a hemodiálise diminui este efeito que podem até desaparecer durante o tratamento, porém esta reação também pode ser associada a reações alérgicas a heparina e/ou resíduos de oxido de etileno, logo deve-se estar atento ao início do sintoma pra identifica-lo. Em todos os casos a aplicação de emolientes tópicos, uso de anti-histamínicos está indicada, em casos específicos o uso de carbonato de cálcio auxilia no alívio dos sintomas.

- Hipertensão pode estar relacionado a ansiedade relacionada ao tratamento, ou excesso de líquidos corpóreos, não é recomentado a administração de hipotensores ou anti-hipertensivos durante a hemodiálise, devendo ser administrado somente se o sintoma persistir após uma hora do término do tratamento ou em casos severos, deve-se compartilhar a conduta com um profissional médico.

- Hipertermia e calafrios está diretamente ligada a imunossupressão, deixando o paciente vulnerável a infecções, pode ser relacionada a pirógenos presentes na solução dialítica causando bacteremia, neste caso, o paciente é afebril antes do início da hemodiálise, apresentando febre logo após o início do tratamento, é indicado a administração de antitérmicos, e a febre assim como tremores desaparecem ao se interromper o procedimento. Em casos de infecções relacionadas ao cateter, o paciente apresenta hipertermia antes de iniciar a hemodiálise, persistente a administração de antitérmicos, é indicado a retirada do cateter e coleta de culturas, em casos de septicemia a hipertermia permanece antes, durante e após o tratamento de hemodiálise, nestas circunstâncias é primordial avaliação constante das inserções de cateteres verificando presença de sinais infecciosos, e em contribuição ao tratamento antitérmico como administração de compressas e medicamentos, 
convém ao enfermeiro monitorar e diminuir se possível a temperatura do dialisador.

Entre os diversos motivos que levam a internação em UTI, Carneiro et al. (2017) em seu estudo aponta a Sepse com a principal causa de internações em UTI, podendo chegar à metade dos pacientes internados. Sepse é uma resposta grave e desregulada à infecção, o choque séptico pode levar a disfunção de vários órgãos, tal fato associa-se ao aumento do tempo de permanência na UTI, neste cenário é comum o desenvolvimento da IRA. O quadro séptico e a instabilidade hemodinâmica compões as prevalentes e principais etiologias da IRA. A Sepse também pode se desenvolver durante a internação neste setor, devido ao grande número de sondas e cateteres necessário durante o tratamento, aumentando o tempo de internação e consequente piora no prognóstico, desta forma é imprescindível o cuidado na inserção, manipulação e manutenção destes cateteres.

O manejo da hemodiálise envolve um contato íntimo com fluidos orgânicos, expondo os trabalhadores a diversos riscos de acidentes de trabalho, tanto químico quanto físico, biológico, e ergonômico, destacando acidentes com perfurocortantes e fluidos corpóreos. A adoção de medidas de biossegurança é imprescindível para prevenção efetiva de acidentes e doenças ocupacionais, assim como a educação e conscientização destes profissionais (SILVIA, 2010).

Amorim et al. (2010), relata que o desempenho da hemodiálise depende da permeabilidade dos cateteres, os circuitos devem permanecer livres de coágulos de sangue e permeáveis garantindo a fluidez do sangue, uma coagulação do sistema é responsável, pela suspensão da terapia dialítica, ou troca do sistema ou capilares, que podem acarretar anemia e consequente necessidade de hemotransfusão. Fatores como coágulos intraluminais, dobras ou má posição, contribuem para coagulação completa ou parcial, ocasionando bloqueio ou redução de fluxo, ou ainda produz ativação de leucócitos e plaquetas no lúmen, propiciando condições para a formação de novos trombos, daí a necessidade do uso de anticoagulantes 
durante a hemodiálise, os principais anticoagulantes utilizados são a heparina, heparina de baixo peso molecular, heparina-protamina e citrato trissódico a 4\%. Não há consenso sobre qual seria o método de anticoagulação ideal para os pacientes em terapias contínuas, diversos fatores contribuem para o alto índice de coagulação dos sistemas de diálise, estando relacionados com o estado clínico ou com a própria técnica e materiais utilizados:

- Heparina é o anticoagulante mais utilizado na atualidade, com vantagens como o baixo custo, meia vida de 6 horas e alta eficiência, pode ser revertida com o uso de protamina e, monitorizada pelo nível de anticoagulação com o tempo parcial de tromboplastina ativado (TTPA), contudo, as desvantagens do seu uso referem-se à anticoagulação sistêmica e a trombocitopenia.

- O citrato trissódico a 4\% é um anticoagulante que age quelando o cálcio sanguíneo, bloqueando algumas etapas da cascata de coagulação que dependem desse íon, o sangue com citrato que retorna ao paciente contém cálcio iônico, que serão metabolizados principalmente pelo fígado, convertendo cada molécula de citrato em três de bicarbonato, motivo pelo qual se dispensa a utilização de solução tampão, contudo o cálcio perdido nesse processo deve ser reposto endovenosamente em infusão concomitante com a hemodiálise, com controle rigoroso dos níveis séricos de cálcio pré e pós filtro periodicamente de acordo com os protocolos institucionais, evitando assim a hipocalemia, hipernatremia, alcalose metabólica e acidose metabólica.

Para Sousa (2015) a capacitação profissional, assim como aprimoramento dos seus desempenhos, permite que a enfermagem conquiste seu espaço, exigindo seus profissionais cada vez mais qualificados para o desempenho profissional, buscando a qualidade da assistência, aprimorando seus conhecimentos, direcionando suas ações o mais cientificamente possível, para prestar o cuidado em sua totalidade do modo mais individual possível, entendendo e respeitando os limites dos pacientes críticos.

RC: 94364

Disponível em: https://www.nucleodoconhecimento.com.br/saude/pacientes-emhemodialise 


\section{CONSIDERAÇÕES FINAIS}

Este estudo evidenciou que a IRA tem causas multifatoriais, sem um consenso exato em sua definição, porém com meios de classificação universais, o que facilita o seu tratamento. O quadro agudo pode ser reversível uma vez que tratado em conjunto com sua causa, desta forma a unidade de terapia intensiva é o ambiente mais favorável para este tratamento.

A sepse é um quadro clínico recorrente em unidade de terapia intensiva, podendo evoluir rapidamente para o choque séptico necessitando de drogas vasoativas para manutenção da homeostasia, sua repercussão é a hipoperfusão dos órgãos, que pode progredir para IRA, assim como outras condições clínicas, podem resultar na insuficiência renal aguda. Uma das atribuições do enfermeiro intensivista neste cenário é estar capacitado para garantir medidas de prevenção desta e outras complicações, bem como acompanhamento constante dos sinais e sintomas de insuficiência renal.

A unidade de terapia intensiva é uma área de assistência a pacientes críticos em risco de morte, requer além de uma infraestrutura, materiais específicos, profissionais especializados, em busca de aprimoramentos profissional constante. $\mathrm{O}$ enfermeiro neste cenário, além de desempenhar um papel importante no gerenciamento dos cuidados, na administração da unidade, gerenciamento da equipe, garantindo materiais e suporte para os mais diversos procedimentos que ocorrem neste setor, ter conhecimento científico e uma avaliação clínica apurada para detectar os primeiros sinais de alterações no quadro clínico do paciente.

O trabalho multiprofissional assegura desde uma dieta adequada, monitorização clínica e laboratorial destes pacientes, manejo adequado de balanço hídrico, inserção de cateteres para hemodiálise quando necessário, controle de perdas através de processos hemodialíticos, cuidados preventivos e de tratamento de infecções, controle sérico de eletrólitos assim como a manutenção, promovendo 
tratamento imediato às alterações do paciente crítico. Por esta razão o enfermeiro intensivista deve ter o domínio da fisiologia, compreender complicações frequentes da hemodiálise e pacientes no processo dialítico.

Conclui-se por tanto que o enfermeiro deve buscar constante aprimoramento e atualização profissional, uma vez que sua atuação interfere diretamente no prognóstico do paciente, além das suas ações e conhecimento refletirem e impulsionarem o trabalho da sua equipe, proporcionando um bom vínculo entre profissionais, paciente e familiares.

\section{REFERÊNCIAS}

AMORIM, Ângelo Mário Vieira et al. Eficácia do citrato como anticoagulante na hemodiálise veno-venosa contínua com ciclo de 60 horas em unidade de terapia intensiva. Conscientiae Saúde, São Paulo, v. 9, n. 2, p. 187-193, fev. 2010. Disponível em: https://www.redalyc.org/articulo.oa?id=92915260004. Acesso em: 06 set. 2020.

BARBOSA, Karina Regiane Almeida et al. INDICAÇÕES DE HEMODIÁLISE DE EMERGÊNCIA EM UMA UNIDADE DE TERAPIA INTENSIVA DE UM HOSPITAL PARTICULAR DA CIDADE DE ATIBAIA - SP. Science In Health, São Paulo, v. 3, n. 3, p. 131-138, 2012. Disponível em: http://arquivos.cruzeirodosuleducacional.edu.br/principal/new/revista_scienceinhealth 109_set_dez_2012/science_03_03_131-138.pdf. Acesso em: 19 maio 2020.

CARNEIRO, Tatiane Aguiar et al. IDENTIFICATION AND RECOVERY OF RENAL FUNCTION IN NON-DIALYTIC PATIENTS IN THE INTENSIVE THERAPY SCENARIO. Reme: Revista Mineira de Enfermagem, [S.L.], v. 21, p. 2-6, 2017. GN1 Genesis Network. http://dx.doi.org/10.5935/1415-2762.20170039. Disponível em: https://cdn.publisher.gn1.link/reme.org.br/pdf/e1029.pdf. Acesso em: 04 set. 2020. 
GRASSI, Mariana de Freitas et al. Diagnósticos, resultados e intervenções de enfermagem em pacientes com lesão renal aguda. Acta Paulista de Enfermagem: Acta Paulista de Enfermagem, São Paulo, v. 5, n. 30, p. 538-545, 24 out. 2017. Mensal. Disponível em: https://www.scielo.br/scielo.php?pid=S010321002017000500538\&script=sci_abstract\&tlng=pt. Acesso em: 21 maio 2020

LEONARDI, Egle. $O$ enfermeiro nos cuidados do paciente em hemodiálise. 2019. Por Egle Leonardi. Disponível em: https://www.posgraduacaoenfermagem.com.br/enfermagem-clinica/38-o-enfermeironos-cuidados-do-paciente-em-hemodialise. Acesso em: 02 set. 2020.

MANUAL DE DIÁLISE DA NEFROCLÍNICA, 2011., 2012, Piauí. Manual de Diálise: Nefrologia - hemodiálise. Piauí: Hgv-Pi, 2012. 80 p. Disponível em: http://www.hgv.pi.gov.br/download/201204/HGV25_43447dbcff.pdf. Acesso em: 02 set. 2020.

MINISTÉRIO DA SAÚDE. Assembleia Legislativa. Constituição (2010). Resolução no 7, de 07 de fevereiro de 2010. RESOLUÇÃO № 7, DE 24 DE FEVEREIRO DE 2010. Ministério da Saúde: Agência Nacional de Vigilância Sanitária. 7. ed. Braslia , DISTRITO FEDERAL: Sistema de Legislação da Saúde, 07 fev. 2010. Brasília. Disponível em: https://bvsms.saude.gov.br/bvs/saudelegis/anvisa/2010/res0007_24_02_2010.html. Acesso em: 21 maio 2020.

NASCIMENTO, Roseli Aparecida Matheus do et al. Conhecimento do enfermeiro para identificação precoce da Injúria Renal Aguda. Revista da Escola de Enfermagem da Usp São Paulo, v. 3, n. 50, p. 399-404, 2016. Disponível em: https://www.scielo.br/scielo.php?script=sci_arttext\&pid=S008062342016000300399\&Ing=en\&tIng=en. Acesso em: 21 maio 2020

PONCE, Daniela et al. Injúria renal aguda em unidade de terapia intensiva: estudo prospectivo sobre a incidência, fatores de risco e mortalidade: estudo prospectivo 
sobre a incidência, fatores de risco e mortalidade. Revista Brasileira de Terapia Intensiva, [s.I.], v. 23, n. 3, p. 321-326, set. 2011. FapUNIFESP (SciELO). http://dx.doi.org/10.1590/s0103-507x2011000300010. Acesso em: 27 maio 2020

SCHWANKE, Alessandra Amaral; DANSKI, Mitzy Tannia Reichembach; PONTES, Letícia; KUSMA, Solena Ziemer; LIND, Jolline. Central venous catheter for hemodialysis: incidence of infection and risk factors. Revista Brasileira de Enfermagem, [S.L.], v. 71, n. 3, p. 1115-1121, maio 2018. FapUNIFESP (SciELO). http://dx.doi.org/10.1590/0034-7167-2017-0047. Acesso em: 18 maio 2020

SILVA, Michele Karla Damacena da. Riscos Ocupacionais em um setor de hemodiálise na perspectiva dos trabalhadores da equipe de enfermagem. Revista Enfermagem: Escola Anna Nery, Rio de Janeiro Rj, v. 2, n. 13, p. 279-286, jun. 2010. Bimestral. Disponível em: http://dx.doi.org/10.1590/S141481452009000200007. Acesso em: 02 set. 2020

SOUSA, Verônica Jesus de. A importância dos cuidados de enfermagem prestados em terapia intensiva a pacientes em processos hemodialíticos venosos contínuos: revisão bibliográfica. Atualiza Saúde: Revista Eletrônica de Atualização Científica, Salvador, v. 1, n. 1, p. 99-108, jan. 2015. Semestral. Disponível em: http://atualizarevista.com.br/article/v1-n1-a-importancia-dos-cuidados-deenfermagem-prestados-em-terapia-intensiva-a-pacientes-em-processoshemodialiticos-venosos-continuos-revisao-de-literatura/. Acesso em: 04 set. 2020.

Enviado: Abril, 2021.

Aprovado: Agosto, 2021. 